\title{
Developmental trends in utilizing perceptual closure for grouping of shape: Effects of spatial proximity and collinearity
}

\author{
BAT-SHEVA HADAD and RUTH KIMCHI \\ University of Haifa, Haifa, Israel
}

\begin{abstract}
In two experiments, visual search was used to study the grouping of shape on the basis of perceptual closure among participants 5-23 years of age. We first showed that young children, like adults, demonstrate an efficient search for a concave target among convex distractors for closed connected stimuli but an inefficient search for open stimuli. Reliable developmental differences, however, were observed in search for fragmented stimuli as a function of spatial proximity and collinearity between the closureinducing fragments. When only closure was available, search for all the age groups was equally efficient for spatially close fragments and equally inefficient for spatially distant fragments. When closure and collinearity were available, search for spatially close fragments was equally efficient for all the age groups, but search for spatially distant fragments was inefficient for younger children and improved significantly between ages 5 and 10. These findings suggest that young children can utilize closure as efficiently as can adults for the grouping of shape for closed or nearly closed stimuli. When the closureinducing fragments are spatially distant, only older children and adults, but not 5-year-olds, can utilize collinearity to enhance closure for the perceptual grouping of shape.
\end{abstract}

The consciously perceived visual world is strikingly different from the unstructured mosaic of intensities and colors that stimulates the retina. Internal processes of perceptual organization have, therefore, been postulated to structure these bits and pieces of visual information into the larger coherent units that we experience as environmental objects. Gestalt psychologists have suggested that perceptual organization is achieved by grouping elements together by virtue of certain properties that are present in the image, including proximity, closure, good continuation, common motion, and similarity (Wertheimer, 1923/1955).

The growing body of research on perceptual organization suggests that not all groupings are equal in their time course, attentional demands, and developmental trajectory (e.g., Behrmann \& Kimchi, 2003; Ben Av \& Sagi, 1995; Kellman, 1996; Kimchi, 1998, 2000; Kimchi, Hadad, Behrmann, \& Palmer, 2005; Kimchi \& Razpurker-Apfeld, 2004; Kovacs, Kozma, Feher, \& Benedek, 1999; Kurylo, 1997; Quinn, Bhatt, Brush, Grimes, \& Sharpnack, 2002; Spelke, 1982). This study focuses on the development

This research was supported partly by a grant from the Research Authority, University of Haifa and partly by Max Wertheimer Minerva Center for Cognitive Processes and Human Performance, University of Haifa. We thank the children and staff of Haifa's schools for their participation and cooperation, Hanna Strominger for programming assistance, Anat Hershkovitz and Ruth Romano for assistance in data collection, and Ruxandra Sireteanu and two anonymous reviewers for their comments. Correspondence concerning this article should be addressed to R. Kimchi, Department of Psychology, University of Haifa, Haifa 31905, Israel (e-mail: rkimchi@research.haifa.ac.il). of the ability to utilize closure and its combination with collinearity and proximity to organize fragmented image contours into shapes.

The Gestalt psychologists noted that perceptual closure plays a crucial role in perceptual organization and, in particular, in determining the shape of an object: "If a line forms a closed, or almost closed, figure, we see no longer merely a line on a homogeneous background, but a surface figure bounded by the line" (Koffka, 1935, p. 150). Several psychophysical studies with adults have documented the role of closure in perceptual organization (e.g., Elder \& Zucker, 1993, 1994, 1998; Kimchi, 2000; Kovacs \& Julesz, 1993; Saarinen \& Levi, 1999). For example, contour detection sensitivity is greater for fragmented closed contours (against a cluttered background) than for open contours (Kovacs \& Julesz, 1993), shape discrimination is more precise for closed contours than for nonclosed contours (Saarinen \& Levi, 1999), and search for a concave target among convex distractors is efficient for closed stimuli but inefficient for open stimuli (Elder \& Zucker, 1993). Elder and Zucker (1993) further showed that target detection time for fragmented stimuli decreases monotonically as the length of the connecting segments increases, supporting the notion of a closure continuum (see also Gillam, 1975).

Several studies have demonstrated the advantage of the combination of closure and collinearity over closure alone. Target search is more efficient when distractors are grouped by closure and collinearity than when grouped by closure alone (Donnelly, Humphreys, \& Riddoch, 1991), and reversals in contrast polarity along bounding contours have a detrimental effect on search efficiency when they 
occur at points of high degree of change in contour direction, but not when they occur at straight contour segments (Spehar, 2002). Of particular relevance to the present article, microgenetic studies of the perceptual organization of line configurations have shown that when gaps occur at the corners of a "square" configuration, the global configuration of spatially close line segments, but not of distant line segments, is primed at brief exposures. When the gaps occur at the center of the "square" lines-namely, separating collinear lines - the global configurations of both spatially close and spatially distant line segments are primed at brief exposures (Kimchi, 2000). These findings suggest that spatial proximity facilitates rapid grouping by closure but grouping by closure and collinearity combined is not affected by spatial proximity.

Research on the development of perceptual organization has focused mainly on the emergence of perceptual organization abilities in infancy. Few studies, however, have directly examined perceptual organization with respect to closure. Studies of infants suggest that in addition to common motion (e.g., Kellman \& Spelke, 1983), young infants respond to good continuation and proximity (e.g., Ghim, 1990; Johnson \& Aslin, 1995, 1996; Kavsek, 2002; Quinn, Brown, \& Streppa, 1997; Smith, Johnson, \& Spelke, 2003; Van Giffen \& Haith, 1984). For example, if edges are not aligned or are separated by a large gap, young infants do not perceive them as connected, even if the surfaces undergo common motion (Johnson \& Aslin, 1995, 1996; Smith et al., 2003). Quinn et al. (1997) reported that 3- and 4-month-old infants parsed two overlapping forms by good continuation and, perhaps, closure. Evidence concerning infants' sensitivity to good continuation comes also from developmental studies of the perception of illusory contours. Csibra (2001) showed that 8-montholds, but not 5-month-olds, perceive the figure formed by illusory contours as having the properties of a real object that can act as an occluding surface, but Kavsek (2002) obtained evidence for the perception of static illusory contours in 4-month-olds when the inducing elements were in close physical proximity. Recently, Gerhardstein, Kovacs, Ditre, and Feher (2004), using Gabor-based stimuli and a contour integration task, found that 3-month-olds are able to rely on good continuation of contour elements, although their ability is far from adult-like, but they appear to be completely insensitive to closure.

Studies of the development of perceptual organization beyond the 2 nd year of age are few and far between, but those that exist indicate that some perceptual organization abilities remain incomplete well into childhood (e.g., Kaldy \& Kovacs, 2003; Kimchi et al., 2005; Kovacs, 2000; Kovacs et al., 1999; Lewis et al., 2004; Rieth \& Sireteanu, 1994; Sireteanu, 2000; Sireteanu \& Rieth, 1992). For example, Kovacs (2000; Kovacs et al., 1999) found a significant improvement in children who were between 5 and 14 years of age in visual spatial integration by using a contour detection task with Gabor-based stimuli. Enns and Girgus (1985) obtained evidence suggesting that kindergartners are sensitive to closure, but Abravanel (1982), examining recognition of completed shapes formed by illusory contours, found that the recognition accuracy of 3-year-olds averaged $60 \%$, and it increased to $100 \%$ by age 5 . We do not know, however, of any study in which the development of the ability to utilize closure or the combination of closure with collinearity and proximity for the perceptual grouping of shape in younger and older children has been examined directly.

This study was designed to fill this gap by comparing the performance of 5- and 10-year-old children and young adults in a visual search task similar to the one used by Elder and Zucker (1993). Participants searched as quickly and accurately as possible for a concave target among a variable number of convex distractors. The basic stimuli were composed of two unconnected line segments (see Figure 1). The line segments were the same for the concave and convex stimuli, but their placement relative to each other differed, bending inward for the concave stimuli and outward for the convex ones. Therefore, the discrimination between target and distractors required grouping of the contour segments into coherent 2-D shapes.

Experiment 1 compared children's and adults' utilization of closure in shape perception by examining performance in this visual search task for open versus closed stimuli. The results demonstrated that for young children, as for adults, search efficiency is high for closed connected
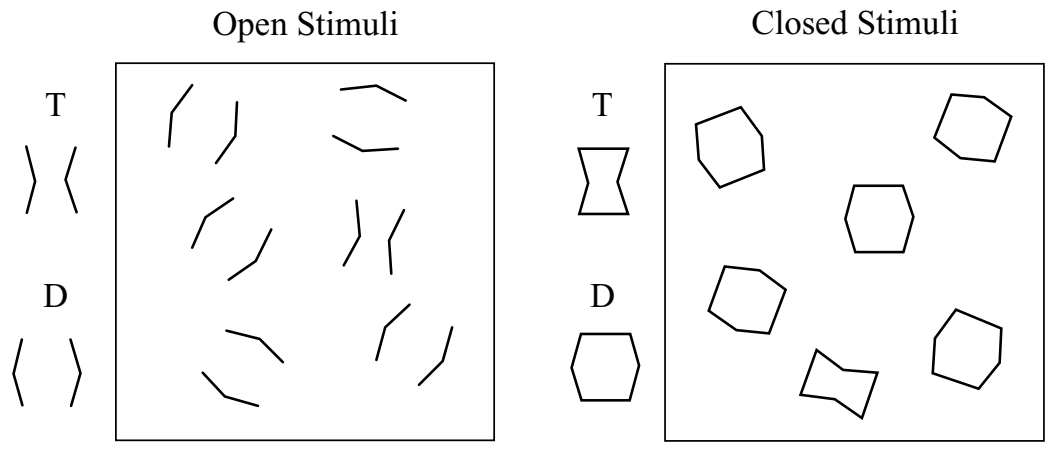

Figure 1. Examples of search displays for the open and the closed stimuli in Experiment 1 . The target (T) and distractors (D) for each example are indicated. The examples illustrate a display size of 6 . 
stimuli but low for open stimuli. In natural scenes, however, closed connected contours often appear in the image as fragmented, due, for example, to occlusion. The perceptual system must group the image fragments and uncover the shape of the object. Previous research with adults suggests that the efficiency of grouping fragmented images into a shape depends on the size of the gaps between the closure-inducing fragments (Elder \& Zucker, 1993) and on the distribution of the gaps along the contour - whether the gaps occur at points of change in contour direction or at straight contour segments (Kimchi, 2000; Spehar, 2002). Accordingly, Experiment 2 compared the ability of children and adults to utilize closure in visual search for fragmented stimuli that vary in the spatial proximity between the closure-inducing line segments and the presence or absence of collinearity.

\section{EXPERIMENT 1}

In this experiment, participants had to detect the presence or absence of a spindle-like (concave) shape among a variable number of barrel-like (convex) shapes that were either open or fully closed (Figure 1). The primary dependent variable was search rate, defined by the slope of the best-fitting linear reaction time (RT) function over the number of items in the display. Target search is considered efficient and effortless if the time taken to detect a target is independent of the number of items in the display. If the time taken to detect the target increases with the number of items in the display, search is considered inefficient and effortful (e.g., Duncan \& Humphreys, 1989; Treisman \& Gormican, 1988).

Previous studies with adults showed fast and efficient search for the closed stimuli and slow and inefficient search for the open ones (Elder \& Zucker, 1993), suggesting that closure plays a critical role in shape perception. This experiment addressed the question of whether children's utilization of closure in shape perception is similar to that of adults.

\section{Method}

Participants. One hundred nineteen participants were tested: forty 5 -year-olds $(M=5.2$, range $=4.11-5.9)$, thirty-nine 10-yearolds $(M=10.6$, range $=9.4-10.8)$, and forty 23 -year-olds $(M=$ 23 , range $=18-30$ ). All the participants had normal or corrected-tonormal vision. All the experiments with children were conducted in kindergartens and schools in the Haifa district and were approved by the University of Haifa Ethical Committee. Informed consent was obtained from the parents after the procedure had been fully explained.

Apparatus. Display presentation and data collection were controlled by a Dell GX-270 PC (adults) or an IBM T21 portable computer (children). The participants responded by pressing on computer keyboard keys (adults) or by moving a joystick upward or downward (children).

Stimuli. The basic stimuli were composed of two unconnected bent lines of equal size, spatially arranged to form a concave, spindle-like shape (the two lines bending inward) and a convex, barrel-like shape (the two lines bending outward). Target and distractor configurations for the open and closed conditions are depicted in Figure 1. In the open condition, the target (the concave shape) and the distractors (the convex shapes) were composed of just the two unconnected lines. In the closed condition, the target and the distractors were closed shapes formed by adding two identical connecting lines at the top and bottom of each of the open figures. The length of the top and bottom connecting lines was identical for the target and the distractors. The stimuli were randomly presented in one of 36 possible orientations. At a viewing distance of $70 \mathrm{~cm}$, each configuration subtended $1.88^{\circ}$ in height and $1.47^{\circ}$ in width (width refers to the distance between the end points of the bent lines). Contour length was identical for the convex and the concave shapes. The distance between the convex inflection points (the convex shape) subtended $1.88^{\circ}$, and the distance between the concave inflection points (the concave shape) subtended $0.98^{\circ}$. Display sizes of 2,6 , or 10 items were used. The items were presented in jittered random locations in a $5 \times 4$ matrix subtending $15.95^{\circ} \times 12.88^{\circ}$.

Design and Procedure. The experiment employed an orthogonal combination of four factors: age $(5,10$, or 23 years), stimulus (open or closed), trial type (target present or absent), and display size (2, 6 , or 10). Age and stimulus were between-subjects factors. Trial type and display size were manipulated within subjects and randomized within block, with each combination occurring on an equal number of trials. There were 72 trials in an experimental block, preceded by 24 practice trials. Considerable practice and feedback were given to ensure that the children understood the task and were not distracted from it. The children were given initial training using cardboard pictures. The experimental block was divided into six subblocks of 12 trials. To increase children's motivation, we used vivid pictures to present a story about a character on a mission (e.g., a monkey trying to reach bananas) and told the children that their own progress in the task would help the character reach its goal.

Each trial started with a central fixation cross presented for $500 \mathrm{msec}$. Following a 500-msec interval, the target display appeared and remained present until response or $7 \mathrm{sec}$ had elapsed.

\section{Results and Discussion}

All summaries and analyses of RT were based on participants' mean RTs for correct responses. Mean RTs and error rates (ERs) for all age groups as a function of display size and stimulus on target-present and target-absent trials are presented in Table 1. The participants in all the age groups were highly accurate (mean ER $=4.8 \%, 1.9 \%$, and $2.5 \%$, for $5-, 10-$, and 23 -year-olds, respectively). A mixed design ANOVA (age $\times$ stimulus $\times$ trial type $\times$ display size) conducted on the ER data showed that young children were less accurate than adults $[F(2,113)=10.19$, $p<.0001]$, and all the participants were less accurate in search for open than for closed stimuli $[F(1,113)=7.29$, $p<.008]$. ER was higher for target-present trials (average, $4.2 \%$ ) than for target-absent trials (average, $2 \%$ ) $[F(1,113)=23.19, p<.0001]$, suggesting that the participants made more misses than false alarms. This effect interacted with age $[F(2,113)=3.89, p<.025]$, with the adults making hardly any false alarms, indicating that the adults were more liberal than the young children in their accuracy criterion in the target-present trials. No other reliable interactions involving age or any other indications of speed-accuracy trade-offs were found.

Two RT measures were examined: baseline RT (RT for a display size of 2), conventionally considered to measure response speed independently of search rate, and the slope of the best-fitting linear function relating RT to display size, conventionally considered to measure search efficiency.

Mean baseline RTs and RT slopes for target-present trials are depicted in Figure 2 as a function of age and stimulus. Our RT analyses focused on target-present data 
Table 1

Mean Reaction Times (RTs, in Milliseconds) and Error Rates (ERs, in Percentages) on Target-Present and Target-Absent Trials in Experiment 1

\begin{tabular}{|c|c|c|c|c|c|c|c|c|c|c|c|c|c|c|c|c|c|c|}
\hline \multirow[b]{4}{*}{ Stimulus } & \multicolumn{18}{|c|}{ Display Size } \\
\hline & \multicolumn{6}{|c|}{ Age 5} & \multicolumn{6}{|c|}{ Age 10} & \multicolumn{6}{|c|}{ Age 23} \\
\hline & \multicolumn{2}{|c|}{2} & \multicolumn{2}{|c|}{6} & \multicolumn{2}{|c|}{10} & \multicolumn{2}{|c|}{2} & \multicolumn{2}{|c|}{6} & \multicolumn{2}{|c|}{10} & \multicolumn{2}{|c|}{2} & \multicolumn{2}{|c|}{6} & \multicolumn{2}{|c|}{10} \\
\hline & RT & ER & RT & ER & RT & ER & RT & ER & RT & ER & RT & ER & RT & ER & RT & ER & RT & ER \\
\hline \multicolumn{19}{|c|}{ Target Present } \\
\hline Open & 1,815 & 5.8 & 2,223 & 7.8 & 2,318 & 2.0 & 1,165 & 1.6 & 1,383 & 2.3 & 1,517 & 5.2 & 694 & 3.2 & 813 & 3.8 & 887 & 9.5 \\
\hline Closed & 1,462 & 3.8 & 1,473 & 5.4 & 1,460 & 4.2 & 1,008 & 1.9 & 987 & 1.2 & 1,007 & 0.4 & 595 & 1.6 & 592 & 2.0 & 641 & 2.8 \\
\hline \multicolumn{19}{|c|}{ Target Absent } \\
\hline Open & 2,018 & 2.3 & 2,662 & 3.2 & 2,963 & 2.6 & 1,328 & 2.4 & 1,785 & 2.0 & 2,112 & 1.8 & 754 & 1.0 & 967 & 0.7 & 1,158 & 0.4 \\
\hline Closed & 1,659 & 3.0 & 1,609 & 4.8 & 1,519 & 2.6 & 1,107 & 1.2 & 1,101 & 1.9 & 1,087 & 0.8 & 638 & 1.6 & 709 & 1.2 & 756 & 1.3 \\
\hline
\end{tabular}

because only target-present trials require a discrimination of the target from the distractors and because target-absent slopes provide less reliable measures of search efficiency because of the variable criteria used for deciding target absence (Chun \& Wolfe, 1996). ${ }^{1}$

A between-subjects ANOVA (age $\times$ stimulus) conducted on baseline RTs (Figure 2A) showed, as was expected, that baseline RTs decreased significantly with age $[F(2,113)=157.69, p<.0001]$. Responses to closed stimuli were faster than those to open ones $[F(1,113)=$ $19.43, p<.0001]$, but this difference did not vary significantly with age $[F(2,113)=2.80, p>.065]$, suggesting no differential developmental changes in target-distractor discriminability for the different stimuli.

The central analysis concerned the RT slopes as an index of search efficiency. This analysis revealed a significant effect of stimulus $[F(1,113)=55.98, p<.0001]$ and a significant interaction between age and stimulus $[F(2,113)=5.42, p<.006]$, indicating age-related changes in search rate that depended on the stimulus involved (Figure 2B). The RT slopes for the closed stimuli were shallow $(-0.3,-0.1$, and $5.8 \mathrm{msec} /$ item for 5,10 , and 23 years, respectively), demonstrating an equally efficient search in all the age groups $(F<1)$. The RT slopes for the open stimuli were considerably steeper, indicating an inefficient search $(63,44$, and $24.2 \mathrm{msec} /$ item for 5,10 , and 23 years, respectively) that improved with age $[F(2,57)=6.48, p<.003]$. Post hoc Duncan comparisons showed a significant difference in RT slopes only between ages 5 and $23(p<.05)$.

These results clearly show that search efficiency for a concave target among convex distractors was high for closed stimuli for all the age groups, whereas search for the open stimuli was inefficient and improved with age. These findings suggest that closure plays a similar role in shape perception for young children and adults. Five- and 10 -year-olds, like adults, are able to derive the shape of a closed figure, although encountering difficulty in doing so when closure is absent. This difficulty was more pronounced in younger children, as is evident in the agerelated improvement in search rates. In the absence of closure (i.e., the open stimuli), the discrimination between the concave and the convex stimuli cannot be based on shape properties but, rather, requires an explicit apprehending of the relative spatial placement of the two line segments for each stimulus. Thus, the age-related improvements for the open stimuli may be a result of improvements in spatial abilities (e.g., Kimchi et al., 2005; Stiles, 2001).
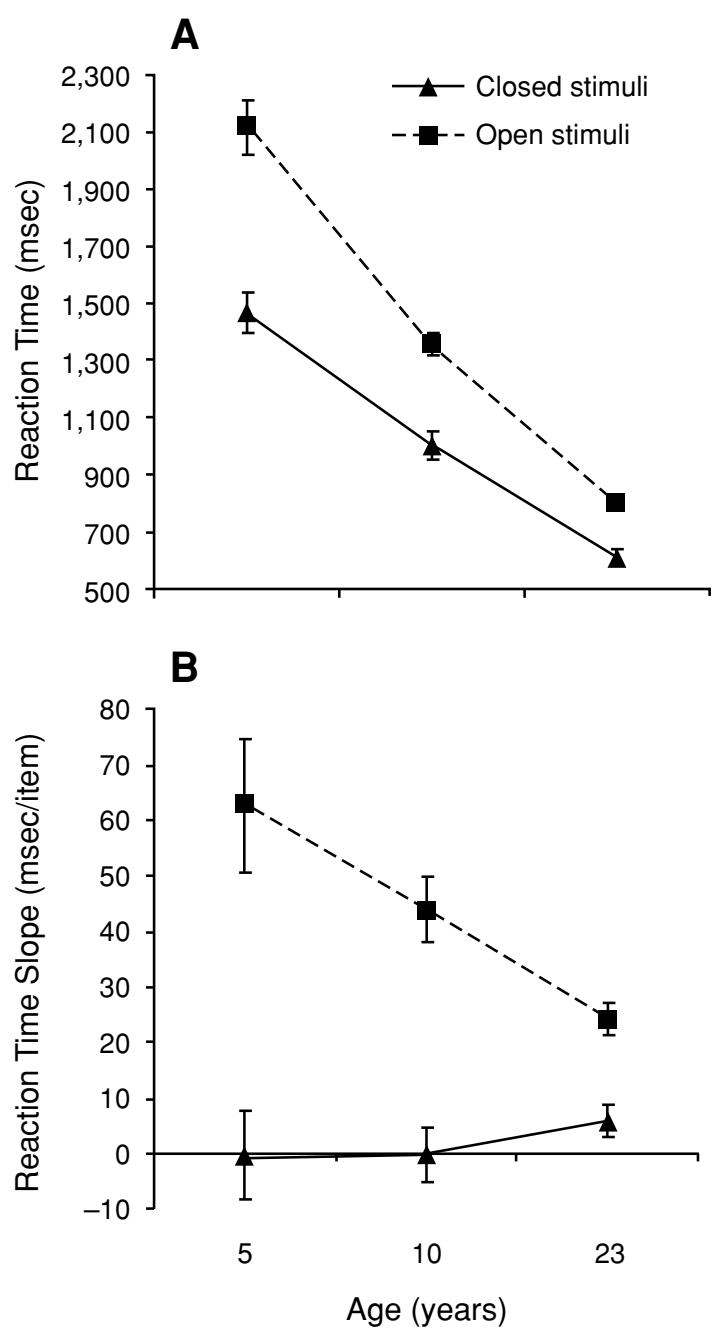

Figure 2. Search results for target-present trials in Experiment 1: (A) Mean baseline reaction times and (B) search slopes for open and closed stimuli as a function of age. 
Having demonstrated that young children are as good as adults in deriving the shape of a closed, connected figure, we now will turn to an examination of the ability of children and adults to utilize closure in fragmented figures as a function of the spatial proximity between the closure-inducing fragments and the presence or absence of collinearity.

\section{EXPERIMENT 2}

This experiment employed the same visual search task as that in Experiment 1: Participants had to detect the presence or absence of a spindle-like (concave) shape among a variable number of barrel-like (convex) shapes. Target and distractors were disconnected line configurations in which the spatial proximity between the line segments varied and, depending on the location of the gaps, either closure alone or closure and collinearity were present (Figure 3 ).

Given Kimchi's (2000) microgenetic results, we expected a differential effect of spatial proximity on adults' search rate for the two types of stimuli. Target search was expected to be efficient for both the closure and the closureand-collinearity stimuli when the closure-inducing line segments were spatially close, but only for the closure-andcollinearity stimuli when the line segments were spatially distant. The critical question, however, concerned agerelated changes as a function of stimuli (closure vs. closure and collinearity) and spatial proximity (small vs. large gap).

\section{Method}

Participants. Two-hundred thirty-nine new participants were tested: seventy-two 5-year-olds $(M=5.2$, range $=4.6-5.10)$, eighty- four 10-year-olds $(M=10.4$, range $=9.6-10.11)$, and eighty-three 23-year-olds $(M=22.9$, range $=20-31)$.

Stimuli. The closed connected concave and convex shapes used in Experiment 1 were adapted to form two types of fragmented stimuli. The closure stimuli were generated by removing the end portions in each of the top and bottom connecting lines, yielding a fragmented figure with four gaps. The closure-and-collinearity stimuli were generated by removing the central portion in each of two connecting lines so that the remaining portions of each connecting line were collinear, yielding a fragmented figure with two gaps. The line segments were either spatially close (small gap condition) or spatially distant (large gap condition). For each stimulus type-closure and closure-and-collinearity stimuli-the size of the line segments was kept the same in the two gap conditions. ${ }^{2}$ The total gap size was the same for the closure and the closure-and-collinearity stimuli for each gap condition. Target and distractor configurations for the four combinations of stimulus and gap are presented in Figure 3. At a viewing distance of $70 \mathrm{~cm}$, the stimuli subtended $1.88^{\circ} \times 1.47^{\circ}$ and $1.88^{\circ} \times 1.97^{\circ}$ for the small-gap and the large-gap conditions, respectively. The gaps between the lines in the closure stimuli subtended $0.24^{\circ}$ each in the small gap condition and $0.49^{\circ}$ each in the large gap condition. The gaps in the closure-and-collinearity stimuli subtended $0.48^{\circ}$ each in the small gap condition and $0.98^{\circ}$ each in the large gap condition. Display sizes of 2,6 , or 10 items were used. The items were presented in jittered random locations in a $5 \times 4$ matrix subtending $15.95^{\circ} \times 12.88^{\circ}$.

Design and Procedure. The experiment employed an orthogonal combination of five factors: age $(5,10$, or 23 years), gap (small or large), stimulus (closure or closure and collinearity), trial type (target present or absent), and display size (2, 6, or 10). Age, gap, and stimulus were between-subjects factors. Trial type and display size were manipulated within subjects and randomized within block, with each combination occurring on an equal number of trials. There were 72 trials in an experimental block, preceded by 24 practice trials. All other aspects of the design and procedure were the same as those in Experiment 1.

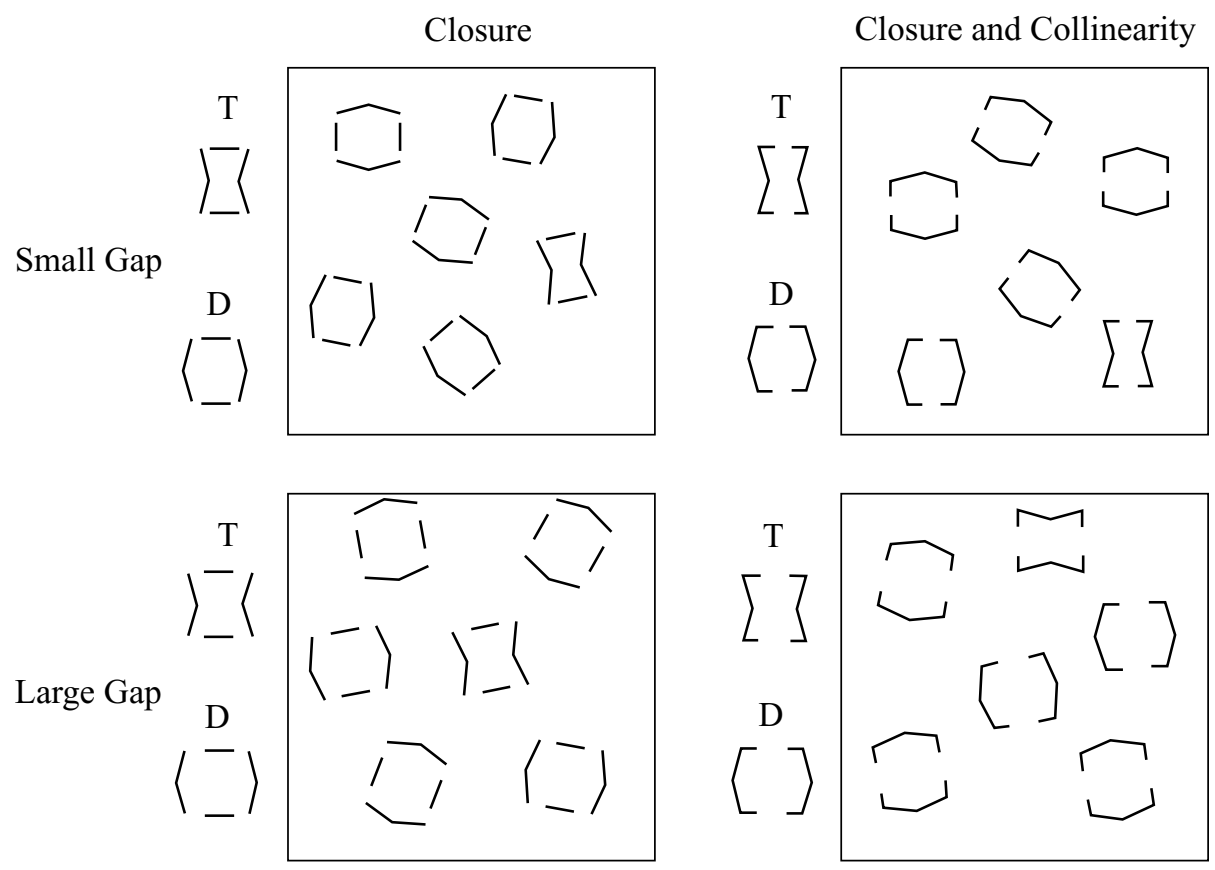

Figure 3. Examples of search displays in Experiment 2. An example is shown for each combination of stimulus (closure or closure and collinearity) and gap (small or large). The target ( $T$ ) and distractors (D) for each example are indicated. The examples illustrate a display size of 6. 


\section{Results and Discussion}

Mean RTs and ERs for all the age groups as a function of display size, stimulus, and gap size for target-present and target-absent trials are presented in Table 2. The participants in all the age groups were highly accurate (mean $\mathrm{ER}=3.3 \%, 1.8 \%$, and $2.3 \%$, for $5-, 10-$, and 23 -yearolds, respectively). A mixed design ANOVA (age $\times$ gap $\times$ stimulus $\times$ trial type $\times$ display size) conducted on the ER data showed that young children were less accurate than adults $[F(2,227)=5.32, p<.006]$. The participants made slightly more misses (average, $2.9 \%$ ) than false alarms (average, $2 \%)[F(1,227)=13.04, p<.0004]$. No interactions involving age were found, and there was no indication of speed-accuracy trade-offs.

Mean baseline RTs and RT slopes for target-present trials are shown in Figure 4 as a function of age, gap, and stimulus. Again, we focused on target-present data, for the reasons reported in Experiment $1 .^{3}$

A between-subjects ANOVA (age $\times$ gap $\times$ stimulus) conducted on the baseline RTs (Figure 4A) showed, as was expected, that baseline RT decreased with age $[F(2,227)=255.84, p<.0001]$. RTs for closure-andcollinearity stimuli were shorter than those for closure stimuli $[F(1,227)=4.37, p<.04]$, but this effect did not vary with age $[F(2,227)=1.51, p>.22]$, suggesting no differential developmental changes in target-distractor discriminability for the different stimuli. No other main effect or interaction was significant.

The analysis of the RT slopes revealed reliable developmental differences in search rates that depended on both stimulus and gap $[F(2,227)=3.14, p<.05]$. As can be seen in Figure 4B, spatial proximity between the line segments had a similar effect on children and adults' search for the closure stimuli, but a different effect on their search for the closure-and-collinearity stimuli. For the closure stimuli, the effect of gap was significant $[F(1,121)=$ $17.2, p<.0001]$ and did not vary with age $(F<1)$. When the gap was small, RT slopes were shallow $(-1,8.8$, and $7.1 \mathrm{msec} /$ item for 5-, 10-, and 23-year-olds, respectively), indicating an equally efficient search in all the age groups $(F<1)$. When the gap was large, RT slopes were considerably steeper $(21,24.2$, and $24.3 \mathrm{msec} / \mathrm{item}$, for 5-, 10-, and 23-year-olds, respectively), indicating an inefficient search that also did not vary with age $(F<1)$. For the closure-and-collinearity stimuli, however, there was a significant effect of gap $[F(1,106)=16.42, p<.0001]$ and a significant interaction between gap and age $[F(2,106)=$ $7.88, p<.0006]$, suggesting age-related changes that depended on gap size. In the case of a small gap, all the age groups exhibited shallow slopes ( $-4.8,2.6$, and $3.5 \mathrm{msec} /$ item for 5-, 10-, and 23-year-olds), indicating an efficient search that did not vary with age $[F(2,51)=1.16, p>$ $.32]$. In the case of a large gap, search rate improved considerably with age $[F(2,55)=7.29, p<.002]$, from an inefficient search at age 5 (slope $=39.7 \mathrm{msec} /$ item) to an efficient search in older age (slope $=11.4$ and $5.6 \mathrm{msec} /$ item, for 10- and 23-year-olds, respectively). Post hoc Duncan comparisons revealed a significant decrease in RT slope between 5 and 10 years of age $(p<.05)$ and no change from age 10 to adulthood.

The results for 10-year-olds and adults showed that the effect of spatial proximity between the closure-inducing line segments on search performance depended on whether closure alone or closure and collinearity were available. When closure alone was present in the stimuli, search for the shape of spatially close line segments was highly efficient, whereas the shape of spatially distant line segments was searched inefficiently. When both closure and collinearity were available, the shape of both spatially close and spatially distant line segments was searched efficiently. The performance of the 5-year-olds, however, depended on spatial proximity between the line segments, regardless of whether closure alone or closure and collinearity were available. Their search was efficient for the shapes of spatially close line segments and inefficient for shapes of spatially distant line segments. Note that the difference in the overall size of the stimuli between the two gap conditions cannot account for the present results, because no

Table 2

Mean Reaction Times (RTs, in Milliseconds) and Error Rates (ERs, in Percentages) on Target-Present and Target-Absent Trials in Experiment 2

\begin{tabular}{|c|c|c|c|c|c|c|c|c|c|c|c|c|c|c|c|c|c|c|}
\hline \multirow[b]{4}{*}{ Stimulus } & \multicolumn{18}{|c|}{ Display Size } \\
\hline & \multicolumn{6}{|c|}{ Age 5} & \multicolumn{6}{|c|}{ Age 10} & \multicolumn{6}{|c|}{ Age 23} \\
\hline & \multicolumn{2}{|c|}{2} & \multicolumn{2}{|c|}{6} & \multicolumn{2}{|c|}{10} & \multicolumn{2}{|c|}{2} & \multicolumn{2}{|c|}{6} & \multicolumn{2}{|c|}{10} & \multicolumn{2}{|c|}{2} & \multicolumn{2}{|c|}{6} & \multicolumn{2}{|c|}{10} \\
\hline & RT & ER & RT & ER & RT & ER & RT & ER & RT & ER & RT & ER & RT & ER & RT & ER & RT & ER \\
\hline \multicolumn{19}{|c|}{ Target Present } \\
\hline CLO-S & 1,753 & 7.3 & 1,718 & 3.1 & 1,747 & 3.0 & 1,104 & 1.3 & 1,128 & 0.3 & 1,174 & 1.0 & 652 & 2.6 & 664 & 1.8 & 708 & 4.0 \\
\hline CLO-L & 1,781 & 3.1 & 1,831 & 3.3 & 1,949 & 4.6 & 1,125 & 4.2 & 1,308 & 1.0 & 1,318 & 1.6 & 705 & 2.5 & 859 & 4.7 & 900 & 7.2 \\
\hline CLO+COL-S & 1,773 & 2.1 & 1,791 & 2.0 & 1,734 & 3.5 & 949 & 2.3 & 909 & 1.2 & 970 & 2.4 & 601 & 1.4 & 619 & 1.5 & 629 & 2.8 \\
\hline CLO+COL-L & 1,758 & 3.8 & 2,121 & 2.9 & 2,076 & 4.2 & 930 & 3.6 & 1,002 & 2.8 & 1,021 & 2.8 & 602 & 4.2 & 622 & 1.3 & 647 & 3.1 \\
\hline \multicolumn{19}{|c|}{ Target Absent } \\
\hline CLO-S & 1,982 & 5.0 & 2,232 & 5.9 & 2,381 & 3.8 & 1,264 & 1.0 & 1,355 & 1.7 & 1,426 & 1.0 & 703 & 1.8 & 837 & 1.2 & 886 & 0.4 \\
\hline CLO-L & 2,155 & 3.7 & 2,469 & 0.3 & 2,757 & 3.4 & 1,368 & 1.9 & 1,832 & 1.3 & 2,199 & 3.1 & 837 & 2.9 & 1,180 & 0.8 & 1,394 & 0.7 \\
\hline CLO+COL-S & 1,942 & 3.5 & 1,921 & 3.1 & 1,955 & 1.0 & 986 & 2.0 & 1,067 & 2.0 & 1,073 & 1.6 & 630 & 2.8 & 668 & 1.5 & 718 & 1.0 \\
\hline CLO+COL-L & 1,960 & 1.9 & 2,192 & 1.4 & 2,528 & 2.3 & 1,018 & 2.6 & 1,140 & 0.8 & 1,232 & 1.2 & 622 & 2.9 & 688 & 0.7 & 741 & 1.7 \\
\hline
\end{tabular}

Note-CLO, closure; CLO+COL, closure and collinearity; S, small gap; L, large gap. 


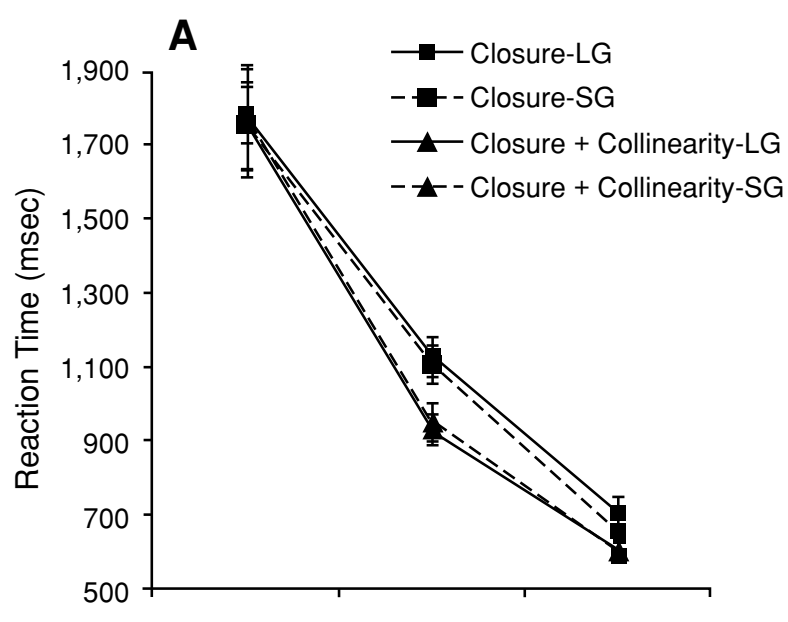

B

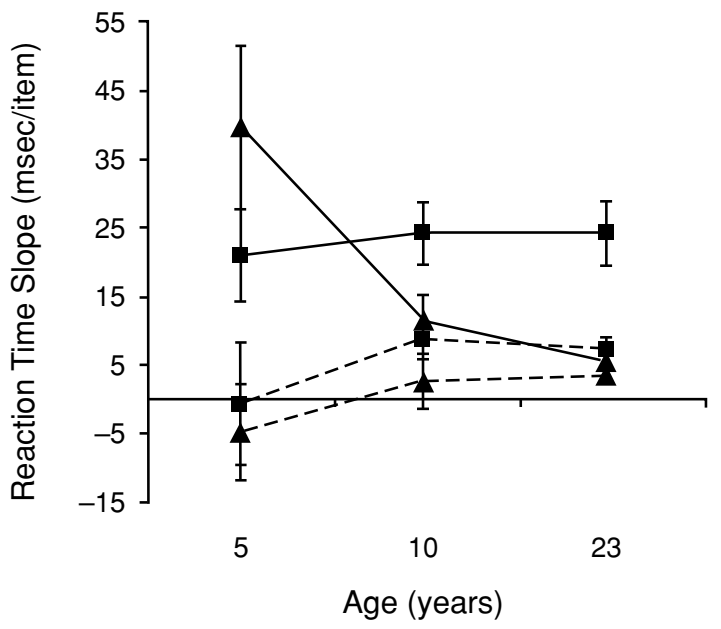

Figure 4. Search results for target-present trials in Experiment 2: (A) Mean baseline reaction times and (B) search slopes for the closure and the closure-and-collinearity stimuli as a function of gap (SG, small gap; LG, large gap) and age.

age-related changes as a function of gap were observed for the closure stimuli. It is only when closure was combined with collinearity that a differential age-related change as a function of gap was observed.

One may argue that it is not the combination of closure with collinearity that accounts for the difference in results for the closure versus the closure-and-collinearity stimuli in the large gap condition but, rather, local corner information. That is, shape discrimination for the closureand-collinearity stimuli was based on the acute and obtuse angles generated by the corners of the concave and convex stimuli, respectively, and since this information was not available for the closure stimuli, target search was inefficient. Our results demonstrating similar efficient search for both types of stimuli in the small gap condition, as well as the results of Elder and Zucker (1993), showing that local corner information does not play an important role in the shape discrimination, are inconsistent with this alter- native account. To further rule it out, we replicated Elder and Zucker's (1993) experiment with the stimuli depicted in Figure 5, in which the corners point outward but the angular difference between the target and the distractors is the same as in the closure-and-collinearity stimuli. ${ }^{4}$

As can be seen in Figure 6, RT slopes for these stimuli were steep (109.1, 113.6, and $94.7 \mathrm{msec} /$ item for 5-, 10-, and 24-year-olds, respectively), indicating an inefficient search that did not vary with age $(F<1)$. These findings clearly show that local corner information cannot account for the search performance. Rather, it is the combination of closure and collinearity that accounts for the difference in search efficiency between the closure-and-collinearity and the closure stimuli that was observed for 10-year-olds and adults in the large gap condition.

Thus, the present results suggest that young children, like adults, can utilize closure for the perceptual grouping of shape when the closure-inducing line segments are spatially close. Only older children and adults, but not 5 -yearolds, are able to use collinearity to enhance closure when the closure-inducing line segments are spatially distant.

Interestingly, search in the small gap conditions in the present experiment (for both the closure and the closureand-collinearity stimuli) was as efficient as search for the closed connected stimuli in Experiment 1, for all the age groups. These findings converge with previous findings suggesting that contrary to Palmer and Rock's (1994) proposal, uniform connectedness may not play a crucial role in early perceptual organization in both adults (Han, Humhreys, \& Chen, 1999; Kimchi, 1998, 2000) and children as young as 5 years old (see also Kimchi et al., 2005).

\section{GENERAL DISCUSSION}

The results of Experiment 1 showed that young children and adults can efficiently search for a concave target among convex distractors for closed, connected stimuli but demonstrate an inefficient search for open stimuli. In natural scenes, however, closed figures are often fragmented, with gaps varying in size, and, depending on the

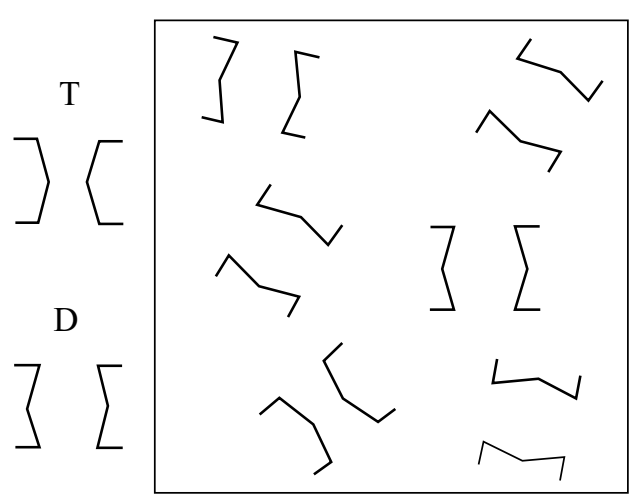

Figure 5. An example of the search displays for the control condition in Experiment 2. 


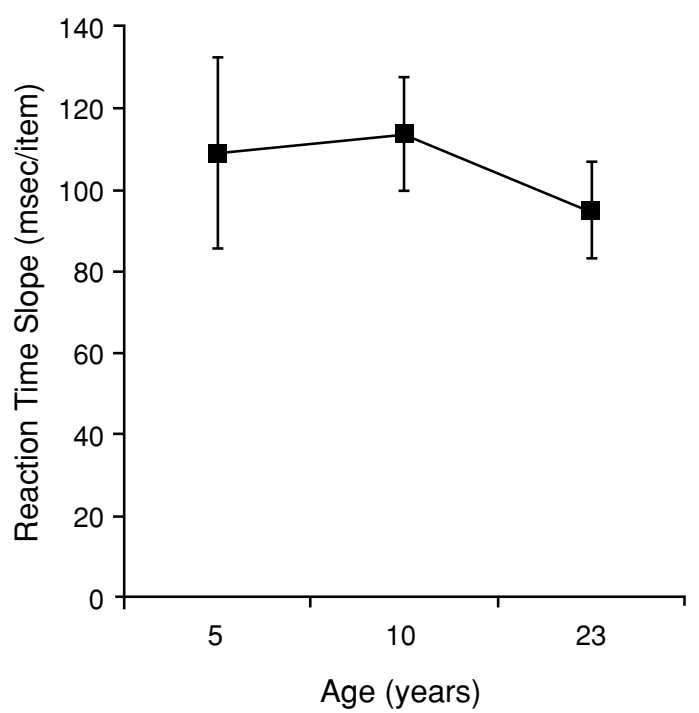

Figure 6. Search slopes for target-present trials in the control condition in Experiment 2 as a function of age.

gaps' distribution along the contour, collinearity may be present in the image. Therefore, the main objective of this study was to examine developmental trends in the ability to utilize closure as a function of the spatial proximity between the closure-inducing fragments and the presence or absence of collinearity. The results of Experiment 2 show that when closure alone was available in the stimuli, the performance of both young children and adults was affected by spatial proximity, and no age-related changes were observed. For all the age groups considered, search for the shape of spatially close line segments was equally efficient, whereas the shape of spatially distant line segments was searched equally inefficiently. When closure and collinearity were available, age trends in search rates differed as a function of the spatial proximity between the closure-inducing line segments. Search for the shape of spatially close lines was equally efficient for all the age groups. However, when the line segments were spatially distant, search was inefficient for the younger children and improved significantly in the transition from ages 5 to 10 .

The search performance of 10 -year-olds and adults indicate that spatial proximity between the closure-inducing line segments influenced grouping into a shape for the closure stimuli, but not for the closure-and-collinearity stimuli. These results converged with microgenetic results in adult observers (Kimchi, 2000), which demonstrated that spatial proximity can facilitate rapid grouping into a shape by closure but that grouping by closure and collinearity combined is not affected by spatial proximity. Other studies also have shown the superiority of the combination of closure and collinearity over closure alone (Donnelly et al., 1991; Spehar, 2002). The present results further demonstrate that the combination of closure and collinearity is particularly powerful for grouping of line segments into a shape when the closure-inducing line segments are not in close proximity.

The search performance of the 5-year-olds, however, appears to depend critically on the spatial proximity between the closure-inducing line segments: It was as efficient as that of older children and adults when the lines were spatially close but was inefficient when the lines were spatially distant, regardless of whether closure alone or closure and collinearity were available.

Taken together, these results indicate that both children as young as 5 years of age and adults can use closure, in an equally efficient manner, for the organization of unconnected line segments into a shape, provided that the closure-inducing line segments are in close spatial proximity. However, when the closure-inducing line segments are spatially distant, yielding relatively weak closure, only older children (i.e., 10-year-olds) and adults can utilize collinearity, when available, to enhance closure for the perceptual grouping of shape.

The finding of a significant improvement in the ability to utilize collinearity for spatially distant line segments between ages 5 and 10 converges with the findings of Kovacs (2000; Kovacs et al., 1999), suggesting a longer developmental progression in the ability to employ long-range contour interpolation. It has been suggested that cortical connectivity in Layers $2 / 3$ of V1 that seem to be immature even at 5 years of age (Burkhalter, Bernardo, \& Charles, 1993), on the one hand, and a delayed development of feedback connections between V1 and V2 (Burkhalter, 1993), on the other, underlie the reduced contour integration ability in young children. These laterdeveloping connections are believed to be part of the physiological mechanism that enables collinearity and, possibly, closure detection (Kovacs, 1996).

Before concluding, it is important to note that our results are generally consistent with the idea that perceptual closure is not an all-or-none phenomenon but, rather, a continuum (Elder \& Zucker, 1993, 1994; Gillam, 1975). Our results, however, are inconsistent with the particular proposal of Elder and Zucker (1994). They proposed a measure of perceptual closure that emphasizes large gaps, relative to small ones (for a total gap size). Thus, according to Elder and Zucker's (1994) proposal, performance for the closure stimuli should be superior to that for the closure-and-collinearity stimuli because there are four smaller gaps in the former versus two larger gaps in the latter (for each gap condition). Our results, however, did not confirm this prediction. In the small gap condition, for all the age groups, search was equally efficient for both the closure and the closure-and-collinearity stimuli. The results for the large gap condition, at least for 10-year-olds and adults, are even more striking: Search was efficient for the closure-and-collinearity stimuli but inefficient for the closure stimuli. These results indicate that large gaps separating collinear lines (thus allowing collinearity to be used, as in the closure-and-collinearity stimuli) have a marginal effect on the efficiency of grouping into a shape, whereas smaller gaps separating noncollinear lines (as in 
the closure stimuli) can have a detrimental effect when the total gap size is rather large.

In sum, we demonstrated that young children can utilize closure as efficiently as adults for the perceptual grouping of shape for closed or nearly closed stimuli. When the closure-inducing fragments are spatially distant, older children and adults, but not 5-year-olds, can utilize collinearity to enhance closure for the perceptual grouping of fragments into a shape. These findings converge with previous findings, both behavioral and neurophysiological, suggesting that the mechanisms underlying long-range contour interpolation are slow to reach functional maturity.

\section{REFERENCES}

Abravanel, E. (1982). Perceiving subjective contours during early childhood. Journal of Experimental Child Psychology, 33, 280-287.

BehrmanN, M., \& KImCHI, R. (2003). What does visual agnosia tell us about perceptual organization and its relationship to object perception? Journal of Experimental Psychology: Human Perception \& Performance, 29, 19-42.

BEN Av, M. B., \& SAGI, D. (1995). Perceptual grouping by similarity and proximity: Experimental results can be predicted by intensity autocorrelations. Vision Research, 35, 853-866.

Burkhalter, A. (1993). Development of forward and feedback connections between areas V1 and V2 of human visual cortex. Cerebral Cortex, 3, 476-487.

Burkhalter, A., Bernardo, K. L., \& Charles, V. (1993). Development of local circuits in human visual cortex. Journal of Neuroscience, 13, 1916-1931.

Chun, M. M., \& Wolfe, J. M. (1996). Just say no: How are visual searches terminated when there is no target present? Cognitive Psychology, 30, 39-78.

CsIBRA, G. (2001). Illusory contour figures are perceived as occluding surfaces by 8-month-old infants. Developmental Science, 4, F7-F11.

Donnelly, N., Humphreys, G. W., \& RidDOCH, M. J. (1991). Parallel computation of primitive shape descriptions. Journal of Experimental Psychology: Human Perception \& Performance, 17, 561-570.

DunCan, J., \& Humphreys, G. W. (1989). Visual search and stimulus similarity. Psychological Review, 96, 433-458.

ELDER, J. [H.], \& ZuCKER, S. [W.] (1993). The effect of contour closure on the rapid discrimination of two-dimensional shapes. Vision Research, 33, 981-991.

EldER, J. [H.], \& ZuCKER, S. [W.] (1994). A measure of closure. Vision Research, 34, 3361-3369.

Elder, J. H., \& ZuCKER, S. W. (1998). Evidence for boundary-specific grouping. Vision Research, 38, 143-152.

EnNs, J. T., \& Girgus, J. S. (1985). Perceptual grouping and spatial distortion: A developmental study. Developmental Psychology, 21, 241-246.

Gerhardstein, P., Kovacs, I., Ditre, J., \& Feher, A. (2004). Detection of contour continuity and closure in three-month-olds. Vision Research, 44, 2981-2988.

GHIM, H. R. (1990). Evidence for perceptual organization in infants: Perception of subjective contours by young infants. Infant Behavior \& Development, 13, 221-248.

Gillam, B. (1975). New evidence for "closure" in perception. Perception \& Psychophysics, 17, 521-524.

Han, S., Humphreys, G. W., \& Chen, L. (1999). Uniform connectedness and classical Gestalt principles of perceptual grouping. Perception \& Psychophysics, 61, 661-674.

Johnson, S. P., \& Aslin, R. N. (1995). Perception of object unity in 2-month-old infants. Developmental Psychology, 31, 739-745.

Johnson, S. P., \& Aslin, R. N. (1996). Perception of object unity in young infants: The roles of motion, depth, and orientation. Cognitive Development, 11, 161-180.

KaLDY, Z., \& Kovacs, I. (2003). Visual context integration is not fully developed in 4-year-old children. Perception, 32, 657-666.
KavseK, M. J. (2002). The perception of static subjective contours in infancy. Child Development, 73, 331-344.

Kellman, P. J. (1996). The origins of object perception. In T. Kit-Fong \& R. Gelman (Eds.), Perceptual and cognitive development (pp. 348). San Diego: Academic Press.

Kellman, P. J., \& Spelke, E. S. (1983). Perception of partly occluded objects in infancy. Cognitive Psychology, 15, 483-524.

KIMCHI, R. (1998). Uniform connectedness and grouping in the perceptual organization of hierarchical patterns. Journal of Experimental Psychology: Human Perception \& Performance, 24, 1105-1118.

KimCHI, R. (2000). The perceptual organization of visual objects: A microgenetic analysis. Vision Research, 40, 1333-1347.

Kimchi, R., Hadad, B.-S., Behrmann, M., \& Palmer, S. E. (2005). Microgenesis and ontogenesis of perceptual organization evidence from global and local processing of hierarchical patterns. Psychological Science, 16, 282-290.

Kimchi, R., \& RAZPURKER-APFeLD, I. (2004). Perceptual grouping and attention: Not all groupings are equal. Psychonomic Bulletin \& Review, 11, 687-696.

KoffKA, K. (1935). Principles of Gestalt psychology. New York: Harcourt Brace Jovanovich.

Kovacs, I. (1996). Gestalten of today: Early processing of visual contours and surfaces. Behavioural Brain Research, 82, 1-11.

Kovacs, I. (2000). Human development of perceptual organization. Vision Research, 40, 1301-1310.

Kovacs, I., \& Julesz, B., (1993). A closed curve is much more than an incomplete one: Effect of closure in figure-ground segmentation. Proceedings of the National Academy of Sciences, 90, 7495-7497.

Kovacs, I., Kozma, P., Feher, A., \& BenedeK, G. (1999). Late maturation of visual spatial integration in humans. Proceedings of the $\mathrm{Na}$ tional Academy of Sciences, 96, 12204-12209.

KurYlo, D. D. (1997). Time course of perceptual grouping. Perception \& Psychophysics, 59, 142-147.

Lewis, T. L., Ellemberg, D., Maurer, D., Dirks, M., Wilkinson, F., \& WiLson, H. R. (2004). A window on the normal development of sensitivity to global form in Glass patterns. Perception, 33, 409-418.

PALMER, S., \& RocK, I. (1994). Rethinking perceptual organization: The role of uniform connectedness. Psychonomic Bulletin \& Review, 1, 29-55.

Quinn, P. C., Bhatt, R. S., Brush, D., Grimes, A., \& Sharpnack, H. (2002). Development of form similarity as a Gestalt grouping principle in infancy. Psychological Science, 13, 320-328.

Quinn, P. C., Brown, C. R., \& StrepPa, M. L. (1997). Perceptual organization of complex visual configurations by young infants. Infant Behavior \& Development, 20, 35-46.

Rieth, C., \& Sireteanu, R. (1994). Texture segmentation and "popout" in infants and children: The effect of test field size. Spatial Vision, 8, 173-191.

SaARINEN, J., \& Levi, D. M. (1999). The effect of contour closure on shape perception. Spatial Vision, 12, 227-238.

Sireteanu, R. (2000). Texture segmentation, "pop-out," and feature binding in infants and children. In L. P. Lipsitt \& C. Rovee-Collier et al. (Eds.), Progress in infancy research (Vol. 1, pp. 183-249). Mahwah, NJ: Erlbaum.

SiREteAnU, R., \& RiEth, C. (1992). Texture segregation in infants and children. Behavioural Brain Research, 49, 133-139.

Smith, W., Johnson, S. P., \& Spelke, E. S. (2003). Motion and edge sensitivity in perception of object unity. Cognitive Psychology, 46, 31-64.

SPehaR, B. (2002). The role of contrast polarity in perceptual closure. Vision Research, 42, 343-350.

SPELKE, E. S. (1982). Perceptual knowledge of objects in infancy. In J. Mehler, M. Garrett, \& E. Walker (Eds.), Perspectives on mental representation (pp. 409-430). Hillsdale, NJ: Erlbaum.

Stiles, J. (2001). Spatial cognitive development. In C.A. Nelson \& M. Luciana (Eds.), Handbook of developmental cognitive neuroscience (pp. 399-414). Cambridge, MA: MIT Press.

Treisman, A., \& Gormican, S. (1988). Feature analysis in early vision: Evidence from search asymmetries. Psychological Review, 95, 15-48.

VAN Giffen, K., \& Haith, M. M. (1984). Infant visual response to Gestalt geometric forms. Infant Behavior \& Development, 7, 335-346. 
Wertheimer, M. (1955). Gestalt theory. In W. D. Ellis (Ed.), A source book of Gestalt psychology (pp. 1-16). London: Routhedge \& Kegan Paul. (Originally published 1923)

\section{NOTES}

1. The target-absent data showed a pattern of results similar to that for the target-present data. The age $\times$ stimulus $\times$ trial type interaction was not significant for baseline RT $(F<1)$, and although this interaction was significant for RT slopes $[F(2,113)=3.22, p<.05]$, a breakdown of this interaction showed no significant age $\times$ trial type interaction either for the open stimulus $[F(2,57)=1.52, p>.23]$ or for the closed stimulus $[F(2,56)=2.52, p>.09]$, indicating similar age trends in target-present and target-absent trials for both stimuli.

2 . This ensured that the two gap conditions differed in spatial proximity between the closure-inducing line segments. Obviously, this resulted in a difference in the overall size of the stimuli between the two gap conditions. If, however, the overall size of the stimuli was kept the same in the two gap conditions, spatial proximity would be confounded with amount of visible information (i.e., the size of the closure-inducing line segments).

3. The target-absent data showed a similar pattern of results; the age $x$ stimulus $\times$ gap $\times$ trial type interaction was not significant $[F<1$ and $F(2,227)=2.84, p>.06$, for baseline RT and RT slopes, respectively]. 4. Thirty-eight new participants, ten 5 -year-olds $(M=5.7$, range $=$ 4.8-5.11), eighteen 10-year-olds $(M=10.7$, range $=9.4-10.11)$, and ten 24-year-olds $(M=24$, range $=20-30)$ were tested in this control condition. Each stimulus subtended $1.88^{\circ} \times 2.61^{\circ}$. All other aspects of the displays, design, and procedure were the same as those in the main experiment.

(Manuscript received August 23, 2005;

revision accepted for publication January 4, 2006.) 